\section{The Electrophoretic Distribution of Protein-bound Carbohydrates During Attacks of Asthma Bronchiale}

In a previous communication ${ }^{1}$, the correlation of protein-bound carbohydrates (PBC) and their electrophoretic distribution to bronchial asthma was examined. Two types of distribution were found, half of our cases showing no significant changes at all, the other half being of the 'albuminotropic type', closely correlated to eosinophilia. These results were obtained from patients while no attack was in progress. We were therefore interested in possible changes in the electropherograms of PBCs in patients during an asthmatic attack, in comparison with the findings between the attacks.

12 typical adult cases with severe asthma bronchiale were examined. The routine laboratory findings were normal (excepted occasional eosinophilia). The electrophoresis of the PBCs and their staining was performed according to KöIw ${ }^{2}$ with our modification. In 10 cases out of 12 , a significant increase of the PBCs in the $\alpha$-2globulins was found. The significance was calculated to our own normal values.

The observed increase in the $\alpha-2$-globulin fraction is of no specificity at all, but has been described (BERNASCONl${ }^{3}$ ) after the administration of ACTH, and does not depend upon the functional integrity of the suprarenal cortex.

Indeed, cases of bronchial asthma of long duration and with severe and frequent attacks show a relative corticoid insufficiency. In our patients, the mean value of neutral 17 -ketosteroids in urine was $7.6 \mathrm{mg} / 24 \mathrm{~h}$ (after application of Prednisone, even lower values were obscrved!).

In conclusion, we found in adult patients with severe asthma bronchialis, a transitory increase of PBCs in the $\alpha-2$-globulins during the asthmatic attack. This could be explained as a result of the stress effect, followed by an output of ACTH. The relatively insufficient suprarenal cortex does not respond adequately and the asthmatic attack occurs.

We did not find an increase of the PBCs in the $\gamma$-globulin fraction; but it must be noted that the method used only reveals quantitative and not qualitative changes.

Attention must be drawn to the possibility that antibodies, containing PBCs and precipitating the attacks, may move together with the $\alpha-2$-globulins, thus increasing their action. This is another explanation of our results.

\section{T. KoLoš and J. KELLEN}

Laboratory of the National Spa Strbské Pleso and Laboratory of the National Spa Nory Smokorec (Crechoslon'akia), February 9, 1959.

\section{Zusammenfassung}

Das elektrophoretische Glykoproteinogramm zeigt während schwerer Anfalle von Asthma bronchiale bei der Mehrzahl der Patienten eine signifikante Zunahme der Glykoproteine in der $\alpha-2$-Globulinfraktion, was als Stresseffekt gedeutet wird. Zudem besteht die Möglichkeit, dass den Anfall auslösende Antikörper selber Glykoproteine enthalten und, da sie mit den $\alpha-2$-Globulinen wandern, für die Zunahme dieser Fraktion verantwortlich sind.

1 J. Kellen and T. Kolos, lixper. 14,375 (1958). (1952)

2 E. Köıw and A. GrönWALL, Scand. J. clin. Lab. Invest. 4, 211

${ }^{3}$ C. Bernasconi, Acta endocrinol $25,50(1957)$.

\section{Der Einfluss verschiedener Psychopharmaka auf den Bluthistamingehalt von Ratten}

Uber den Histamingehalt des Rattenblutes ist wenig bekannt, doch wird angenommen, chass er, verglichen mit anderen Tierspexies, ziemlich hoch sei. EMmeLin ${ }^{1}$ fand $0,1-0,33 \mu \mathrm{g} / \mathrm{ml}$ im llasma, HALPERN vicl weniger $(0,036 \mu \mathrm{g} / \mathrm{ml})$. ROSE und BRowNe ${ }^{3}$ fanden im Gesamtblut jedoch nur $0,035-0,00 \mu \mathrm{g} / \mathrm{ml}$.

Wir haben mit der kolorimetrischen Mikromethode von Lowny et al, ${ }^{*}$ das Gesamthistamin im Vollblut und im Plasma von katten nach Extraktion mit Trichloressigsäure bestimmt und in Parallelversuchen mit der biologischen Methode von Con: ${ }^{5}$ am Meerschweinchendarm verglichen. Im lollblut fanden wir bei jüngeren Ratten $(150-200 \mathrm{~g}) 0,185+0,051 \mathrm{~kg} / \mathrm{ml}$ Histamin, bei älteren Ratten $(250-350 \mathrm{~g}) 0,236 \pm 0,022 \mu \mathrm{g} / \mathrm{ml}$ in guter (bereinstimmung beider Methoden. Das Plasma der jungen Tiere enthielt davon einen überraschend grossen Anteil von $0,071 \neq 0,0046 \mu \mathrm{g} / \mathrm{ml}$ Histamin. Da alle Thrombo. zyten durch $10 \mathrm{~min}$ Zentrifugieren bei $500 \mathrm{~g}$ und $10 \mathrm{~min}$ bei $1500 \mathrm{~g}$ entfernt wurden und die Thrombozyten der Ratten verschwindend wenig Histamin enthalten, kann dieser relativ hohe Anteil nicht durch Thrombozyten verursacht sein. Die quantitativen Verhältnisse sind daher denjenigen des Hundeplasmas $(0,04-0,07 \mu \mathrm{g} / \mathrm{ml}$, Barsoum und Gandum ${ }^{6}$ ) ähnlich. Die Leukozyten scheinen ziemlich viel Histamin zu enthalten.

Die Wirkung der verschiedenen Pharmaka wurde an Rattengruppen von je $16-18$ Tieren $(150-200 \mathrm{~g}) \mathrm{im}$ Zeitpunkt der stärksten Wirkung untersucht. Ias Blut wurde durch Halsschnitt entnommen, in einem Gefäss mit Oxalat aufgefangen und sofort 2 mal wite angegeben zentrifugiert. Nach 30 min Extraktion mit $30_{0}^{\circ}$ Trichloressigsäure wurde nochmals während $20 \mathrm{~min}$ mit $1500 \mathrm{~g}$ zentrifugiert und der iberstehende l'asmaextrakt mit der kolorimetrischen Methode nach Lown untersucht.

Burch Narkotika wurde der Histamingehalt im Ilasma im Toleranzstadium unterschiedlich beeinflusst. Einstundige ithernarliose senkte ihn auf $75_{0}^{\circ}$ (Tabelle), während Chloroformmarkose wirkungslos blieb.

Typische Tranquilizer wie Reserpin oder Chlorpromazin verursachen eine deutliche Frhöhung um 14-25\% $\mathrm{o}$. I)a in unseren Versuchen der zeitliche Verlauf nicht untersucht wurde, kann der l'nterschied zwischen beiden Pharmaka rein \%ufällig sein. Auch $1 \mathrm{~h}$ nach Numal ist Histamin im Plasma leicht erhöht.

Psychotomimetische Stoffe können den Histamingehalt erhöhen oder senken. Iproniazid hemmt nicht nur die Monoaminoxydase, sondern auch die I laminoxydase (ZHLLer ef al. ${ }^{\text {? }}$, SCHAYER ${ }^{8}$ ) und damit den Histaminabbau. Dadurch, viclleicht aber auch durch erhöhte Histaminfreisetzung aus dem (rewebe, wird der hohe blutgehalt nach $24 \mathrm{~h}\left(+44^{\circ}\right)$ erklär. Was deutlich erregende Amphetamin hat nach 5 heine viel geringere Steigerung

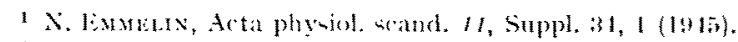

2 B. N. Halpers, Histomin', Cha fammlation Sympumm

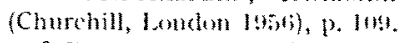

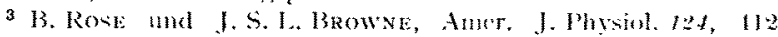
$(1, n+3)$.

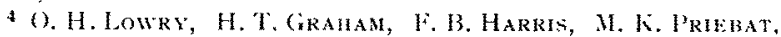
A. R. MARks Und R. I. BREGMAN, J. pharm. exp. Therap. 112, 116 $(1954)$.

5 C. I. Cone, J. Phymidel. s9, 257 (1937).



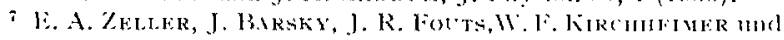

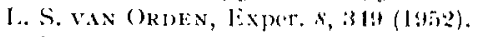

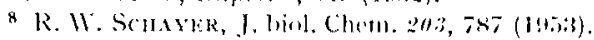

